\title{
SINGULAR CASE OF PAIRS OF BILINEAR, QUADRATIC, OR HERMITIAN FORMS*
}

\author{
BY \\ L. E. DICKSON
}

The main object of this paper is a treatment of the equivalence of pairs of bilinear forms in the singular case by a purely rational method. The problem was first discussed by Kronecker, $\uparrow$ who employed the irrational canonical form due to Weierstrass for the auxiliary non-singular case, instead of the rational canonical form (13) employed here. It is then a simple matter to deduce in Parts II. and III the criteria for the equivalence of pairs of symmetric or Hermitian bilinear forms, or quadratic or Hermitian forms, in the singular case.

\section{PAIRS OF BILINEAR FORMS IN THE SINGULAR CASE}

Let $\phi$ and $\psi$ be bilinear forms in the same variables $x_{1}, \cdots, x_{r}, y_{1}, \cdots, y_{\text {. }}$ with coefficients in a field $F$, such that $\phi$ and $\psi$ are not equivalent in $F$ to forms both of which involve fewer than $r$ variables $x_{i}$ or fewer than $s$ variables $y_{i}$. We shall treat the singular case in which either $r \neq s$, or else $r=s$ and the determinant of $f=u \phi+v \psi$ is zero identically in $u$ and $v$. If $r \neq s$, we take $r>s$ without loss of generality, since we may interchange the letters $x$ and $y$.

Write $f_{i}, \phi_{i}, \psi_{i}$ for the partial derivatives of $f, \phi, \psi$ with respect to $x_{i}$. The linear functions $f_{1}, \cdots, f_{r}$ of $y_{1}, \cdots, y_{0}$ are linearly dependent. Hence there exist homogeneous polynomials $A_{i}$ in $u$ and $v$ of the same degree with coefficients in $F$, such that the $A_{i}$ are not all zero identically and such that $\sum A_{i} f_{i} \equiv 0$ identically in $u, v, y_{1}, \cdots, y_{s}$.

Lemma 1. The degree of $A_{1}, \cdots, A_{r}$ in $u$ and $v$ is not changed if we apply to $f$ non-singular linear transformations on the $x$ 's and the $y$ 's separately whose coefficients are independent of $u$ and $v$.

*Presented to the Society, December 26, 1924; received by the editors in July, 1926.

$\dagger$ Sitzungsberichte der Akademie der Wissenschaften, Berlin, 1890, pp. 1225-37. His brief paper was elaborated by Muth, Elementartheiler, 1899, pp. 93-133. Some minor errata in Muth's work were reproduced in the account in the Encyclopedie des Sciences Mathematiques, tome I, vol. II, 1911, pp. 412-25. 


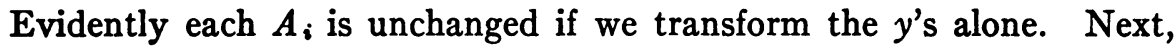
let $f$ become $g$ when the $x$ 's are expressed linearly in terms of new variables $X_{i}$. Then

$$
X_{i}=\sum_{i=1}^{r} a_{j i} x_{i}, f_{i}=\sum_{j=1}^{r} \frac{\partial g}{\partial X_{i}} \frac{\partial X_{i}}{\partial x_{i}}=\sum_{j=1}^{r} a_{j i} g_{i},
$$

and $\sum B_{i} g_{j} \equiv 0$, where $B_{i}=\sum_{i} a_{j i} A_{i}$ is of the same degree as the $A_{i}$.

Of all relations $\sum A_{i} f_{i} \equiv 0$, select one

$$
\sum_{i=1}^{r} A_{i} f_{i} \equiv \sum_{\alpha=0}^{m}(-1)^{\alpha} C_{\alpha} u^{\alpha} v^{m-\alpha} \equiv 0
$$

in which the $A_{i}$ are not all zero identically and are of minimal degree $m$ in $u$ and $v$, while

$$
C_{\alpha}=\sum_{i=1}^{r} c_{\alpha i} f_{i} \quad(\alpha=0,1, \cdots, m),
$$

where the $c_{\alpha i}$ are numbers not all zero of the field $F$ and are independent of $u$ and $v$. By Lemma $1, m$ is an invariant of the pair $\phi$ and $\psi$ under all nonsingular linear transformations on the $x$ 's and the $y$ 's separately.

Lemma 2. $f_{1}, \cdots, f_{r}$ are linearly independent in $F$.

For, if $\sum t_{i} f_{i} \equiv 0$, where the $t_{i}$ are in $F$ and are not all zero, the insertion of $f_{i}=u \phi_{i}+v \psi_{i}$ gives

$$
\sum t_{i} \phi_{i} \equiv 0, \quad \sum t_{i} \psi_{i} \equiv 0 .
$$

Let $t_{c} \neq 0$ and apply the non-singular transformation

$$
x_{0}=t_{0} X_{\bullet}, \quad x_{j}=X_{j}+t_{j} X_{c} \quad(j=1, \cdots, r ; j \neq e) .
$$

Then $\phi=\sum x_{i} \phi_{i}$ becomes

$$
\sum_{j \neq e} X_{j} \phi_{i}+X_{\cdot} \sum_{i=1}^{r} t_{i} \phi_{i}
$$

which lacks $X_{0}$ since the final sum is zero. Likewise, $\psi$ lacks $X_{\bullet}$. But this contradicts our initial assumption.

COROLlary. The invariant $m$ exceeds zero.

For, if $m=0$, (1) becomes $C_{0}=0$ and (2) implies that every $c_{\alpha i}$ is zero, contrary to hypothesis.

Lemaa 3. $C_{0}, \cdots, C_{m}$ are linearly independent in $F$. 
Suppose that $a_{0}, \cdots, a_{m}$ are numbers, not all zero, of $F$ such that

$$
\sum_{\alpha=0}^{m} a_{\alpha} C_{\alpha} \equiv 0 .
$$

Denote the first and second sums in (1) by $Q$ and $R$. Write

$$
P=\sum_{\alpha=0}^{m}(-1)^{\alpha} a_{\alpha} u^{m-\alpha_{v} \alpha} .
$$

Each $P A_{i}$ is a polynomial in $u$ and $v$ of degree $2 m$; let $k_{i}$ be the coefficient of $u^{m} v^{m}$. The terms $\sum k_{i} u^{m} v^{m} f_{i}$ of $P Q$ are obtained from the terms $\sum a_{\alpha} C_{\alpha} u^{m v^{m}}$ of $P R$ by replacing $C_{\alpha}$ by its value (2). Hence (3) implies $\sum k_{i} f_{i} \equiv 0$. By Lemma 2, each $k_{i}=0$. Hence $P Q \equiv 0$ may be written in the form

$$
v^{m+1} \sum f_{i} g_{i}+u^{m+1} \sum f_{i} h_{i} \equiv 0 \quad(i=1, \cdots, r),
$$

in which the $g_{i}$ and $h_{i}$ are polynomials of degree $m-1$ in $u$ and $v$. If they were all zero identically, every $P A_{i}$ and hence every $A_{i}$ would be zero identically. Replacing $f_{i}$ by its value $u \phi_{i}+v \psi_{i}$, we see that the first sum in (5) is of degree $\leqq m$ in $u$, while the second sum is evidently of degree $>m$ in $u$. Hence each sum is zero identically, and one of them gives a linear relation between $f_{1}, \cdots, f_{r}$ the degree in $u$ and $v$ of whose coefficients is $m-1$, while those coefficients $\left(g_{i}\right.$ or $\left.h_{i}\right)$ are not all zero identically. But this contradicts the definition of $m$ as the minimal degree.

By Lemmas 2 and 3, the matrix of the coefficients in (2) has a nonvanishing determinant of order $m+1$. Hence we can annex further rows, also composed of numbers of $F$, and obtain a square matrix

$$
\left(c_{\alpha \beta}\right) \quad(\alpha=0,1, \cdots, r-1 ; \beta=1, \cdots, r)
$$

whose determinant is not zero. Let the non-singular transformation

$$
x_{\beta}=\sum_{\alpha=0}^{r-1} c_{\alpha \beta} X_{\alpha} \quad(\beta=1, \cdots, r),
$$

whose coefficients are independent of $u$ and $v$, replace $f, \phi, \psi$ by $F, \Phi, \Psi$. The partial derivatives of the latter with respect to $X_{i}$ are denoted by $F_{i}, \Phi_{i}, \Psi_{i}$. Then

$$
F_{\alpha}=\sum_{\beta=1}^{r} \frac{\partial f}{\partial x_{\beta}} \frac{\partial x_{\beta}}{\partial X_{\alpha}}=\sum_{\beta=1}^{r} c_{\alpha \beta} f_{\beta}=C_{\alpha},
$$

by (2). By (1),

$$
\sum(-1)^{\alpha} F_{\alpha} u^{\alpha} v^{m-\alpha} \equiv 0
$$


Replacing $F_{\alpha}$ by $u \Phi_{\alpha}+v \Psi_{\alpha}$, and equating to zero the coefficients of $v^{m+1}$, $u v^{m}, u^{2} v^{m-1}, \cdots, u^{m} v, u^{m+1}$, we get

$$
\Psi_{0} \equiv 0, \Psi_{1} \equiv \Phi_{0}, \Psi_{2} \equiv \Phi_{1}, \cdot \cdot, \Psi_{m} \equiv \Phi_{m-1}, \Phi_{m} \equiv 0 .
$$

Hence

$$
F_{0} \equiv u \Psi_{1}, F_{1} \equiv u \Psi_{2}+v \Psi_{1}, \cdots, F_{m-1} \equiv u \Psi_{m}+v \Psi_{m-1}, F_{m} \equiv v \Psi_{m} .
$$

Multiply the first $k$ equations by $v^{k-1},-u v^{k-2}, \cdots,(-u)^{k-2} v,(-u)^{k-1}$, respectively, and add; we get

$$
-(-u)^{k} \Psi_{k} \equiv \sum_{i=0}^{k-1} F_{i}(-u)^{i} v^{k-i-1} \quad(k=1, \cdots, m) .
$$

If $\Psi_{1}, \cdots, \Psi_{m}$ satisfy a linear relation whose coefficients are in the field and are not all zero, multiply it by $u^{m}$ and apply (9). We get a linear relation between $F_{0}, \cdots, F_{m-1}$ whose coefficients are polynomials, not all identically zero, of degree $m-1$ in $u$ and $v$. Inserting the values (7) and applying (2), we get a similar linear relation between $f_{1}, \cdots, f_{r}$, contrary to the definition of $m$. Hence $\Psi_{1}, \cdots, \Psi_{m}$ are linearly independent in the field. We may therefore choose linear functions $Y_{m+1}, \cdots, Y$, of $y_{1}, \cdots, y_{8}$ with coefficients in the field $F$ such that the $\Psi$ and $Y$ are linearly independent in $F$. Then

$$
\eta_{\alpha}=\Psi_{\alpha} \quad(\alpha=1, \cdots, m), \quad \eta_{t}=Y_{t} \quad(t=m+1, \cdots, s)
$$

define a non-singular linear transformation on $y_{1}, \cdots, y_{s}$ with coefficients in $F$. Transformation (6) replaces $u \phi+v \psi$ by

$$
u \Phi+v \Psi=\sum_{i=0}^{r-1} u \Phi_{i} X_{i}+\sum_{i=0}^{r-1} v \Psi_{i} X_{i} .
$$

For $i=0, \cdots, m$, change $i$ to $\alpha-1$ in the first sum, and $i$ to $\alpha$ in the second. For $i>m$, change $i$ to $p$. Then by (8) and (10),

$$
u \Phi+v \Psi=\sum_{\alpha=1}^{m}\left(u X_{\alpha-1}+v X_{\alpha}\right) \eta_{\alpha}+\sum_{p=m+1}^{r-1}\left(u \Phi_{p}+v \Psi_{p}\right) X_{p} .
$$

Here $\Phi_{p}$ and $\Psi_{p}$ are linear in $y_{1}, \cdots, y_{s}$ and hence in $\eta_{1}, \cdots, \eta_{\mathbb{8}}$ by (10):

$$
\Phi_{p}=\sum_{\alpha=1}^{s} a_{p a} \eta_{\alpha}, \quad \Psi_{p}=\sum_{\alpha=1}^{s} b_{p \alpha} \eta_{\alpha}(p=m+1, \cdots, r-1),
$$

where the $a$ 's and $b$ 's are numbers in $F$. We have 


$$
\begin{aligned}
u \Phi+v \Psi & =u\left(X_{0}+\sum_{p} a_{p 1} X_{p}\right) \eta_{1}+u \sum_{\alpha=2}^{m} X_{\alpha-1} \eta_{\alpha} \\
+ & u \sum_{\alpha=2}^{m} \sum_{p} b_{p \alpha-1} X_{p} \eta_{\alpha}+v \sum_{\alpha=1}^{m}\left(X_{\alpha}+\sum_{p} b_{p \alpha} X_{p}\right) \eta_{\alpha}+\rho+\sigma,
\end{aligned}
$$

where the summations with respect to $p$ are from $m+1$ to $r-1$, and $\rho=u\left(l_{2} \eta_{2}+\cdots+l_{m} \eta_{m}\right)$, the $l$ 's being linear in the $X_{p}(p>m)$, while $\sigma$ is a bilinear form in the $X_{p}, \eta_{q}(p>m, q>m)$. In deriving (11), the terms involving $\eta_{\alpha}(\alpha>m)$ which arose from $\Psi_{p}$ were carried to $\sigma$; the terms involving $\eta_{\alpha}(\alpha=2, \cdots, m)$ which arose from $\Phi_{p}$ were carried to $\rho$, while those involving $\eta_{\alpha}(\alpha>m)$ were carried to $\sigma$. Finally we added the terms in the third sum in the second member and subtracted the same from $p$. To (11) apply the non-singular transformation

$$
\begin{aligned}
\xi_{0}=X_{0}+\sum_{p} a_{p 1} X_{p}, \quad \xi_{\alpha}=X_{\alpha}+\sum_{p} b_{p \alpha} X_{p}, \quad \xi_{p}=X_{p} \\
(\alpha=1, \cdots, m ; p=m+1, \cdots, r-1) .
\end{aligned}
$$

We get (12) in which $u \Phi+v \Psi$ is written for $\sigma$.

THEOREM 1. If $\phi$ and $\psi$ are bilinear forms in $x_{1}, \cdots, x_{r}, y_{1}, \cdots, y_{0}$ with coefficients in a field $F$, and $r \geqq s$, and when $r=s$ the determinant $|u \phi+v \psi|$ is zero identically in $u$ and $v$, there exist non-singular linear transformations on the $x$ 's and on the $y$ 's separately with coefficients in $F$ which reduce $u \phi+v \psi$ to

$$
S=\sum_{\alpha=1}^{m}\left(u \xi_{\alpha-1}+v \xi_{\alpha}\right) \eta_{\alpha}+u \sum_{\alpha=2}^{m} l_{\alpha} \eta_{\alpha}+u \Phi+v \Psi,
$$

where $l_{2}, \cdots, l_{m}$ are linear forms in $\xi_{m+1}, \cdots, \xi_{r-1}$, and $\Phi$ and $\Psi$ are bilinear forms in $\xi_{m+1}, \cdots, \xi_{r-1}, \eta_{m+1}, \cdots, \eta_{s}$, all with coefficients in $F$.

If $r<s, f=u \phi+v \psi$ can be reduced to a form derived from (12) by interchanging the letters $\xi$ and $\eta$, since the form derived from $f$ by interchanging the letters $x$ and $y$ can be reduced to (12).

Given a pair of bilinear forms $\lambda$ and $\mu$ with coefficients in $F$ such that $u \lambda+v \mu$ is of rank $R$, we can select independent linear combinations $\phi$ and $\psi$ of $\lambda$ and $\mu$ with coefficients in $F$ such that the greatest common divisor of all $R$-rowed determinants of $u \phi+v \psi$ is not divisible by $u$, whence $\psi$ is of rank $R$. In the corresponding form (12), let $\tau$ be the rank of $u \Phi+v \Psi$.

LEMMa 4. The greatest common divisor of all $\tau$-rowed determinants of $u \Phi+v \Psi$ is not divisible by $u$. 
In the matrix $M$ of (12), the first $m$ columns form the matrix $M_{1}$ of the coefficients of $\xi_{0}, \cdots, \xi_{r-1}$ in

$$
\frac{\partial S}{\partial \eta_{1}}=u \xi_{0}+v \xi_{1}, \frac{\partial S}{\partial \eta_{2}}=u \xi_{1}+v \xi_{2}+u l_{2}, \cdots, \frac{\partial S}{\partial \eta_{m}}=u \xi_{m-1}+v \xi_{m}+u l_{m} .
$$

Since these are evidently linearly independent, $M_{1}$ is of rank $m$. The last $s-m$ columns of $M$ form the matrix $M_{2}$ of the coefficients of $\xi_{0}, \cdots, \xi_{r-1}$ in the partial derivatives of $u \Phi+v \Psi$ with respect to $\eta_{m+1}, \cdots, \eta_{s}$. Hence by the definition of $\tau$, the rank of $M_{2}$ is $\tau$. Thus not every $(m+\tau)$-rowed determinant of $M$ is zero. But every determinant with more than $m+\tau$ columns is zero, since it contains more than $\tau$ columns of $M_{2}$. Hence the rank of $M$ is $m+\tau=R$. Every non-vanishing $(m+\tau)$-rowed determinant of $M$ contains exactly $\tau$ columns of $M_{2}$ and is a linear function of $\tau$-rowed determinants of $M_{2}$ and hence of $u \Phi+v \Psi$. If all $\tau$-rowed determinants of the latter were divisible by $u$, the same would be true of all determinants with $m+\tau=R$ columns of $M$ and hence of $S$, contrary to the remark before Lemma 4.

First, let $u \Phi+v \Psi$ fall under the non-singular case, so that it has the same number of variables in the two series and its determinant is not zero identically in $u$ and $v$. By Lemma 4, this determinant is not divisible by $u$, whence $|\Psi| \neq 0$. Then there exist non-singular transformations on the variables of the two series separately, with coefficients in $F$, which reduce $\Phi$ and $\Psi$ to the respective forms*

$$
\rho=\sum_{k}\left(\sum_{i=1}^{a_{k}-1} x_{k i} y_{k i+1}+x_{k a k} \sum_{i=1}^{a_{k}} b_{k i} y_{k i}\right), \quad \sigma=\sum_{k} \sum_{i=1}^{a_{k}} x_{k i} y_{k i} .
$$

The transformations employed leave unaltered each variable occurring in the first sum in (12) and replace the second sum by $u \sum t_{\alpha} \eta_{\alpha}$, where $t_{\alpha}$ is a linear function of the $x_{k i}$. Hence if $r \geqq s, \phi$ and $\psi$ may be reduced rationally to

$$
J=\sum_{\alpha=1}^{m} \xi_{\alpha-1} \eta_{\alpha}+\sum_{\alpha=2}^{m} \sum_{k} \sum_{i=1}^{a_{k}} d_{k i \alpha} x_{k i} \eta_{\alpha}+\rho, \quad K=\sum_{\alpha=1}^{m} \xi_{\alpha} \eta_{\alpha}+\sigma .
$$

Second, let $u \Phi+v \Psi$ fall under the singular case. We apply to it our initial discussion of $u \phi+v \psi$ and conclude that it can be transformed ration-

*Dickson, Modern Algebraic Theories, Chicago, Sanborn, 1926, p. 116, Theorem IV. A direct verification follows from the fact that the invariant factors other than 1 of $\rho-\lambda \sigma$ are

$$
\lambda^{a k}-\sum_{i=1}^{a k} b_{k i} \lambda^{i-1}
$$$$
(k=1,2, \cdots),
$$

which may be identified with any assigned polynomials. 
ally into a form $R_{1}+u P_{1}+f_{1}$ of type (12) or a form $S_{1}+u Q_{1}+f_{1}$ obtained from it by interchanging the letters $\xi$ and $\eta$, where

$$
\begin{aligned}
f_{1}=u \Phi_{1}+v \Psi_{1}, \quad R_{1} & =\sum_{\alpha=1}^{m_{1}}\left(u \xi_{1 \alpha-1}+v \xi_{1 \alpha}\right) \eta_{1 \alpha}, \quad P_{1}=\sum_{\alpha=2}^{m_{1}} P_{1 \alpha} \eta_{1 \alpha}, \\
S_{1} & =\sum_{\alpha=1}^{n_{1}}\left(u \eta_{1 \alpha-1}+v \eta_{1 \alpha}\right) \xi_{1 \alpha}, \quad Q_{1}=\sum_{\alpha=2}^{n_{1}} q_{1 \alpha} \xi_{1 \alpha} .
\end{aligned}
$$

If $f_{1}$ falls under the non-singular case, $\Phi_{1}$ and $\Psi_{1}$ can be transformed rationally into forms of type (13). If $f_{1}$ falls under the singular case, another repetition of the initial discussion shows that $f_{1}$ can be transformed rationally into $R_{2}+u P_{2}+f_{2}$ or $S_{2}+u Q_{2}+f_{2}$, where $f_{2}, R_{2}, \cdots, Q_{2}$ are of the type $f_{1}, R_{1}, \cdots, Q_{1}$ with the first subscripts 1 changed to 2 .

This process must terminate, since the numbers of variables in $\Phi, \Phi_{1}, \ldots$ form a series of decreasing positive integers.

To prove by induction that $\sum l_{\alpha} \eta_{\alpha}$ (in (12)), $P_{1}$ or $Q_{1}, P_{2}$ or $Q_{2}$, etc., can be removed by a linear transformation with coefficients in $F$ which does not alter the sum of the remaining terms, we assume that all but $\sum l_{\alpha} \eta_{\alpha}$ have been so removed and shall prove that we can then remove it also.

We first show that $R_{1}, S_{1}, R_{2}, \ldots$ can all be written as terms of $u \rho+v \sigma$ in (13) by employing new values of $k$. To do this for $R_{1}$, write

$$
\xi_{1 \alpha}=x_{k \alpha+1}\left(\alpha=0,1, \cdots, m_{1}\right), \eta_{1 \alpha}=y_{k \alpha+1} \quad\left(\alpha=1, \cdots, m_{1}\right),
$$

and $m_{1}=a_{k}-1$. Then $R_{1}$ becomes

$$
u \sum_{i=1}^{a k-1} x_{k i} y_{k i+1}+v \sum_{i=1}^{a k} x_{k i} y_{k i}
$$

for $y_{k 1}=0$; the coefficients of $u$ and $v$ are the terms of (13) for the present value of $k$ and with every $b_{k i}=0$. In $S_{1}$ write

$$
\xi_{1 \alpha}=x_{k_{1}+1-\alpha}\left(\alpha=1, \cdots, n_{1}\right), \eta_{1 \alpha}=y_{k_{1+1-\alpha}} \quad\left(\alpha=0,1, \cdots, n_{1}\right),
$$

and $n_{1}=a_{k}-1$. Then $S_{1}$ becomes (15) for $x_{k a_{k}}=0$.

Whether $u \Phi+v \Psi$ falls under the singular or non-singular case, we may therefore reduce the initial pair $\phi, \psi$ with $r \geqq s$ to a pair (14) such that, for certain values of $k$, either $x_{k a_{k}}$ is suppressed or $y_{k 1}$ is suppressed and every $b_{k i}$ replaced by zero in (13) and the second sum in (14).

It remains to prove that we may take every $d$ to be zero in (14). We shall first prove that we may replace $d_{k i \alpha}$ by zero when $i<a_{k}$. We proceed by induction on $\alpha$, keeping $k$ fixed. For a given integer $n$ such that $2 \leqq n \leqq m$, 
assume that $d_{k i \alpha}=0$ for $i<a_{k}, \alpha=2, \cdots, n-1$. Apply the transformation*

$$
\xi_{n}=X_{n}+\sum_{i=2}^{a_{k}} t_{k i} x_{k i}, \quad y_{k i}=Y_{k i}-t_{k i} \eta_{n} \quad\left(i=2, \cdots, a_{k}\right)
$$

which leaves unaltered the variables not occurring in the left members. The increment to $K$ is zero, while that to $J$ is

$$
\eta_{n+1} \sum_{i=2}^{a_{k}} t_{k i} x_{k i}-\sum_{i=1}^{a_{k}-1} t_{k i+1} x_{k i} \eta_{n}+c x_{k a_{k}} \eta_{n},
$$

where the first sum is to be omitted if $n=m$. Taking

$$
t_{k i+1}=d_{k i n} \quad\left(i=1, \cdots, a_{k}-1\right),
$$

we obtain forms of type (14) in which $d_{k i \alpha}=0$ for $i<a_{k}, \alpha=2, \cdots, n$. Hence the induction is complete and we now have

$$
J=\sum_{\alpha=1}^{m} \xi_{\alpha-1} \eta_{\alpha}+\sum_{\alpha=2}^{m} \sum_{k} e_{k \alpha} x_{k a_{k}} \eta_{\alpha}+\rho, K=\sum_{\alpha=1}^{m} \xi_{\alpha} \eta_{\alpha}+\sigma .
$$

We shall prove that we may replace each $e_{k \alpha}$ by zero. No proof is needed when $k$ is such that $x_{k a_{k}}$ is to be suppressed. We proceed by induction on $\alpha$, keeping $k$ fixed. For a given positive integer $n(n<m)$, assume that $c_{k \alpha}=0$ for $\alpha=m, m-1, \cdots, n+2$.

First, let $n<a_{k}$ and apply the transformation

$$
\begin{aligned}
\xi_{n-i} & =X_{n-i}+t x_{k a k-i} \quad(j=0, \cdots, n), \\
y_{k a k-j} & =Y_{k a k-j}-t \eta_{n-j} \quad(j=0, \cdots, n-1),
\end{aligned}
$$

which leaves unaltered the remaining variables. The increment to $K$ is zero, while that to $J$ cancels to

$$
t x_{k a k} \eta_{n+1}-t x_{k a k} \sum_{j=0}^{n-1} b_{k a k-j} \eta_{n-j} .
$$

Taking $t=-e_{k n+1}$, we obtain (16) with $e_{k \alpha}=0(\alpha=m, \cdots, n+1)$, but with a new term $c x_{k a_{k}} \eta_{1}$. The latter is removed by the transformation $\xi_{0}=$ $\Xi_{0}-c x_{k a_{k}}$.

Second, let $k$ be one of the values for which $y_{k 1}$ is not to be suppressed from (13) and let $n \geqq a_{k}$. Apply transformation (17) with $j=0, \cdots, a_{k}-1$ in both parts. Then $K$ is unaltered, while the increment to $J$ cancels to

*But let the summation extend only to $a_{k}-1$ if $k$ is such that $x_{k a_{k}}$ is to be suppressed from (13) and (14). Then the text holds with $c=0$. 
(18) in which now $j$ takes the values $0, \cdots, a_{k}-1$. We proceed as in the first case.

In either case we obtain forms of type (16) in which now $e_{k \alpha}=0$ ( $\alpha=$ $m, m-1, \cdots, n+1)$. By induction on $n$, we obtain (16) in which every $e_{k \alpha}=0$.

Third, let $k$ be a value for which $y_{k 1}$ is to be suppressed from (16), while every $b_{k i}=0$. Then $m<a_{k}$, whence $n<a_{k}$ and the second case is excluded. In the proof, we write

$$
S=u J+v K, \Sigma_{\alpha}=\frac{\partial S}{\partial \xi_{\alpha}} \quad(\alpha=0, \cdots, m), \quad S_{k i}=\frac{\partial S}{\partial x_{k i}}\left(i=1, \cdots, a_{k}\right) .
$$

By (16) and (13),

(19) $\quad \Sigma_{0}=u \eta_{1}, \quad \Sigma_{\alpha}=u \eta_{\alpha+1}+v \eta_{\alpha} \quad(\alpha=1, \cdots, m-1), \quad \Sigma_{m}=v \eta_{m}$,

the solution of which is

$$
u^{\alpha+1} \eta_{\alpha+1}=\sum_{j=0}^{\alpha}(-1)^{i} u^{\alpha-i} i^{j} \Sigma_{\alpha-i} \quad(\alpha=0, \cdots, m-1) .
$$

Eliminating the resulting value of $\eta_{m}$ from the last equation (19), we get

$$
u^{m} \Sigma_{m}=\sum_{i=1}^{m}(-1)^{i-1} u^{m-i} i^{i} \Sigma_{m-i}
$$

Next,

$$
S_{k i}=u y_{k i+1}+v y_{k i}\left(i<a_{k}\right), S_{k a_{k}}=u \sum_{\alpha=2}^{m} e_{k \alpha} \eta_{\alpha}+v y_{k a_{k}} .
$$

The former with $y_{k 1}$ suppressed gives

$$
u^{i+1} y_{k i+2}=\sum_{j=0}^{i}(-1)^{i} u^{i-j_{v} i} S_{k i+1-i}\left(i=0, \cdots, a_{k}-2\right) .
$$

By (20) and (23), $u^{t} \eta_{t}$ and $u^{t} y_{k}{ }_{t+1}$ are linear functions of the $\Sigma^{\prime}$ 's and $S^{\prime}$ s whose coefficients are polynomials in $u$ and $v$ of degree $t-1$. Multiply the last equation (22) by $u^{m-1}$. If $m \geqq a_{k}$, we get

$$
u^{m-1} S_{k a_{k}}=\sum_{\alpha=2}^{m} e_{k \alpha} u^{m-\alpha} u^{\alpha} \eta_{\alpha}+v u^{m-a_{k}} u^{a_{k}-1} y k a_{k} .
$$

By the remark just made, this yields a linear relation between the first derivatives of $S$ whose coefficients are polynomials in $u$ and $v$ of degree $m-1$. But the $S$ on the left does not occur on the right. This contradicts the definition of $m$. 
This completes the proof that we may take every $d$ to be zero in (14). In stating the following theorem, we keep separate the forms $R_{i}$ and $S_{i}$ previously combined with $u \rho+v \sigma$. For each $i$ only one of the pair $R_{i}$ and $S_{i}$ is a component of the canonical form. We shall change the notation of the variables occurring in a component $S_{j}$.

TheOREM 2. Let $\phi$ and $\psi$ be bilinear forms with coefficients in a field $F$ such that $\psi$ has* the same rank as $f=u \phi+v \psi$. In the singular case, $f$ can be reduced by non-singular linear transformations with coefficients in $F$ to $S=\sum R_{i}$ $+\sum S_{i}+u \rho+v \sigma$, where $\rho$ and $\sigma$ are given by (13), and

$$
R_{i}=\sum_{\alpha=1}^{m_{i}}\left(u \xi_{i \alpha-1}+v \xi_{i \alpha}\right) \eta_{i \alpha}, \quad S_{j}=\sum_{\alpha=1}^{n_{i}}\left(u Y_{j \alpha-1}+v Y_{j \alpha}\right) X_{j \alpha} .
$$

No variable appears in two of the forms $R_{1}, R_{2}, \cdots, S_{1}, S_{2}, \cdots, u \rho+v \sigma$, while all their variables are linearly independent in $F$.

We shall now prove that the invariant factors other than 1 of the matrix $M$ of $S$ coincide with those of $u \rho+v \sigma$. The determinant $D$ of order $d$ of the latter is not zero identically in $u$ and $v$. Write $t=\sum m_{i}+\sum n_{j}$. Then the rank of $M$ will be shown to be $d+t$. In $M$, the coefficient of a product $\xi \eta$ is placed at the intersection of the row $[\xi]$ corresponding to $\xi$ and the column $[\eta]$ corresponding to $\eta$. Similarly for $X Y$.

First, delete the rows $\left[\xi_{i m_{i}}\right]$ corresponding to the $\xi$ 's with maximal second subscripts in the $R_{i}$, and the columns $\left[Y_{j n_{j}}\right]$ corresponding to $Y$ 's with maximal second subscripts in the $S_{j}$. We obtain a square matrix which (apart from elements of $D$ ) has every diagonal element equal to $u$, and has zeros as elements to the right (left) of these $u$ 's in the case of $R_{i}\left(S_{i}\right)$, whence its determinant is $u^{t} D$.

Second, delete the rows $\left[\xi_{i 0}\right]$ and the columns $\left[Y_{j 0}\right]$. We obtain a square matrix which (apart from elements of $D$ ) has every diagonal element equal to $v$, and has zeros as elements to the left (right) of these $v$ 's in the case of $R_{i}\left(S_{i}\right)$, whence its determinant is $v^{t} D$.

Hence the greatest common divisor of all $(d+t)$-rowed determinants of $M$ is $D$. This yields $\dagger$

LEMma 5. The invariant factors other than unity of $S$ coincide with those of $u \rho+v \sigma$.

Hence $\rho$ and $\sigma$ are uniquely determined by the invariant factors of $u \phi+v \psi$.

*We saw that this property may be secured by passing to suitably chosen linear combinations of $\phi$ and $\psi$ with coefficients in $F$.

†See the writer's Modern Algebraic Theories, pp. 96, 97. 
The variables $\xi, X, x$ of the first series in $S$ are linearly independent; likewise for the variables $\eta, Y, y$ of the second series (Theorem 2). Write $W=u \rho+v \sigma$. No variable is common to two of the $R_{i}, S_{i}, W$. Hence a linear function $[S]$ of the first derivatives of $S=W+\sum R_{i}+\sum S_{j}$ with respect to the variables of either series is a sum of linear functions $[W]$, $\left[R_{1}\right],\left[R_{2}\right], \cdots,\left[S_{1}\right], \cdots$ of the first derivatives of $W, R_{1}, R_{2}, \cdots$ with respect to their own variables only. Also, $[S]=0$ implies $[W]=0,\left[R_{1}\right]=$ $0, \cdots$. Since $|W|$ is not identically zero, the vanishing of $[W]$ implies that its coefficients are all zero.

If we suppress the first subscript $i$ from $m_{i}$ and the variables in $R_{i}$, and write $\Sigma_{\alpha}$ for $\partial R_{i} / \partial \xi_{\alpha}$, we have (19)-(21). Since $\Sigma_{0}, \cdots, \Sigma_{m-1}$ are linearly independent, every linear relation between the $\Sigma$ 's is obtained by multiplying (21) by a function of $u$ and $v$.

The first derivatives of $R_{i}$ with respect to the $\eta_{i \alpha}$ are evidently linearly independent. Since $R_{i}$ becomes $S_{i}$ by replacing $\xi, \eta, m$ by $Y, X, n$, we have

LEMma 6. As a complete set of independent linear relations between the first derivatives of $S$ with respect to the variables $\xi, X, x$ of the first series we may take

$$
u^{m_{i}} \frac{\partial S}{\partial \xi_{i m_{i}}}=\sum_{j=1}^{m_{i}}(-1)^{i-1} u^{m_{i}-j_{v} j} \frac{\partial S}{\partial \xi_{i m_{i-j}}},
$$

where $i$ takes the same values as the subscript of $R_{i}$. Here $m_{i}$ is the minimal degree in $u$ and $v$ of the coefficients in any linear relation between the first derivatives of $S$ with respect to the variables $\xi_{i}$ of $R_{i}$. Similarly, $n_{j}$ is the minimal degree in $u$ and $v$ of the coefficients in any linear relation between the first derivatives of $S$ with respect to the variables $Y_{j}$ of $S_{j}$ and these relations of minimal degrees form a complete set of independent linear relations between the first derivatives of $S$ with respect to the variables $\eta, Y, y$ of the second series.

These minimal degrees $m_{i}$ and $n_{j}$ uniquely determine the $R_{i}$ and $S_{j}$. By Lemma 1, they have the same value for $u \phi+v \psi$ as for the canonical form $S$. They are called the minimal numbers of $u \phi+v \psi$ or of $S$.

By Lemmas 5 and 6, and the remarks just following them, we conclude that two pairs of bilinear forms $\phi, \psi$ and $\lambda, \mu$ in $F$, each satisfying the initial assumption and having the property about rank in Theorem 2 , have the same canonical form and hence are equivalent in $F$ if and only if they have the same minimal numbers and the same invariant factors.

Our next step is to prove this result when the two restrictions on the forms are removed.

Let $f$ and $g$ be any bilinear forms with coefficients in $F$ in the singular case. After supplying zero coefficients, if necessary, we may assume that 
there is the same number $n$ of variables $X_{i}$ as variables $Y_{i}$. If $\tau$ is the rank of $h=u f+v g$, there are exactly $n-\tau$ independent linear relations between the first derivatives of $h$ with respect to $X_{1}, \cdots, X_{n}$ (or $Y_{1}, \cdots, Y_{n}$ ). Let them be chosen so that their coefficients are of the least possible degrees $M_{1}, \cdots, M_{n-\tau}$ ( or $N_{1}, \cdots, N_{n-\tau}$ ) in $u$ and $v$. Let the $M$ 's be arranged in order of increasing magnitude; likewise the $N$ 's. The resulting numbers $M_{i}$ and $N_{i}$ are called the minimal numbers of the pair $f, g$.

For example, let $f=X_{1} Y_{3}+X_{3} Y_{1}, g=X_{2} Y_{3}+X_{3} Y_{2}$. Then $h$ has the matrix

$$
\left(\begin{array}{lll}
0 & 0 & u \\
0 & 0 & v \\
u & v & 0
\end{array}\right)
$$

of rank 2. Hence $v h_{1}-u h_{2} \equiv 0$ is the single linear relation between the first partial derivatives $h_{1}, h_{2}, h_{3}$, of $h$ with respect to $X_{1}, X_{2}, X_{3}$. The minimal numbers are $M_{1}=1, N_{1}=1$. Since the two-rowed determinants include $-u^{2}$ and $-v^{2}$, their greatest common divisor is 1 . Hence the matrix has no invariant factor other than 1.

If the first $\rho$ of the $M$ 's and the first $\sigma$ of the $N$ 's are zero, while the others are positive, we can transform $f, g$ into a pair $\phi, \psi$ involving only $r=n-\rho$ variables $x_{i}$ and only $s=n-\sigma$ variables $y_{i}$ (Lemma 2$)$. Then $\phi, \psi$ satisfy our initial assumption that they cannot be transformed rationally into a pair of forms both of which involve fewer than $r$ variables $x_{i}$ or fewer than $s$ variables $y_{i}$.

Since the $M_{i}$ and $N_{i}$ are invariant under every linear transformation on the $X$ 's or on the $Y$ 's (Lemma 1), the positive ones of $M_{i}$ and $N_{i}$ coincide with the above $m_{i}$ and $n_{i}$ of $\phi$ and $\psi$.

Introduce the linear combinations

$$
\phi^{\prime}=a \phi+b \psi, \psi^{\prime}=c \phi+d \psi \quad(a d-b c=1) .
$$

Write $\chi=u \phi+v \psi, \chi^{\prime}=u^{\prime} \phi^{\prime}+v^{\prime} \psi^{\prime}$. Then $\chi=\chi^{\prime}$ if

$$
u^{\prime}=d u-c v, \quad v^{\prime}=-b u+a v .
$$

Before Lemma 4, we chose $a, b, c, d$ so that the greatest common divisor of all $R$-rowed determinants of $\chi^{\prime}$ is not divisible by $u^{\prime}$, where $R$ is the rank of $\chi$ and hence of $\psi^{\prime}$.

Let $\lambda, \mu$ be another pair of bilinear forms in $x_{1}, \cdots, x_{r}, y_{1}, \cdots, y_{\text {, }}$ with coefficients in $F$, having the same minimal numbers and same invariant factors as the pair $\phi, \psi$. Using the same $a, b, c, d$ as in (27), write

$$
\lambda^{\prime}=a \lambda+b \mu, \mu^{\prime}=c \lambda+d \mu, \nu=u \lambda+v \mu, \nu^{\prime}=u^{\prime} \lambda^{\prime}+v^{\prime} \mu^{\prime} .
$$


Since $\chi^{\prime}$ and $\nu^{\prime}$ are transformed into $\chi$ and $\nu$ by (28), they have the same minimal numbers and same invariant factors, and in particular the same rank $R$ and the same greatest common divisor of their $R$-rowed determinants. Hence also $\mu^{\prime}$ is of rank $R$.

Theorem 2 and the remarks following Lemmas 5 and 6 show that $\chi^{\prime}$ and $\nu^{\prime}$ have the same canonical form and hence are equivalent in $F$. Thus the pairs $\phi, \psi$ and $\lambda, \mu$ are equivalent in $F$. This completes the proof of the fundamental

THEOREM 3. In the singular case, two pairs of bilinear forms in the same $n+n$ variables with coefficients in any field $F$ are equivalent in $F$ if and only if they have the same invariant factors and the same minimal numbers.

The canonical pair shows that these may be given any assigned values such that each invariant factor divides the next.

\section{PAIRS OF SYMmetric OR Hermitian BILINEAR FORMS} IN THE SINGULAR CASE

In order to treat simultaneously symmetric and Hermitian bilinear forms, we employ $\tilde{a}$ to denote $a$ or the conjugate imaginary to $a$ in the respective cases. Consider the form

$$
f=\sum_{i, j=1}^{n} a_{i j} x_{i} y_{j}, \quad a_{j i}=\tilde{a}_{i j},
$$

whose matrix $A=\left(a_{i j}\right)$ has the property $A^{\prime}=\tilde{A}$. Then

If

$$
\frac{\partial f}{\partial x_{i}}=\sum_{j=1}^{n} a_{i j} y_{j}, \frac{\partial f}{\partial y_{i}}=\sum a_{j i} x_{j}=\sum_{j=1}^{n} \tilde{a}_{i j} x_{j} .
$$

$$
\sum_{i=1}^{n} c_{i} \frac{\partial f}{\partial x_{i}} \equiv 0
$$

where the $c_{i}$ are independent of the $x$ 's and $y$ 's, then

$$
\sum_{i=1}^{n} c_{i} a_{i j}=0 \quad(j=1, \cdots, n),
$$

whence $\sum \tilde{c}_{i} \partial f / \partial y_{i} \equiv 0$. Take $f=u \phi+v \psi$. Hence each minimal number $M_{i}$ of $\phi, \psi$ coincides with a minimal number $N_{i}$ and vice versa.

Consider two pairs of forms $\phi, \psi$ and $\lambda, \mu$ whose $n$-rowed matrices $A, B$, $C, D$ have the property $M^{\prime}=\tilde{M}$. Let $u \phi+v \psi$ and $u \lambda+v \mu$ have the same minimal numbers $M_{i}$ (and hence the same $N_{i}$ ) and the same invariant factors. Then the pairs are equivalent by Theorem 3, so that there exist non-singular $n$-rowed matrices $P$ and $Q$ for which

$$
C=P A Q, \quad D=P B Q \text {. }
$$


Hence* there exists a non-singular $n$-rowed matrix $E$ such that

$$
C=E^{\prime} A \tilde{E}, \quad D=E^{\prime} B \tilde{E},
$$

whence the linear transformation on the $x$ 's with the matrix $E$ and that on the $y$ 's with the matrix $\tilde{E}$ replace $\phi$ by $\lambda$ and $\psi$ by $\mu$. Since this argument may be reversed, we have

THEOREM 4. In the singular case, two pairs of symmetric (or Hermitian) bilinear forms are equivalent under a non-singular linear transformation on the $x$ 's and the cogredient (or conjugate) transformation on the $y$ 's if and only if they have the same invariant factors and the same minimal numbers $M_{i}$ (which here coincide with the $N_{i}$ ).

In seeking a canonical pair for such a pair $\phi, \psi$, note that the number of the $M_{i}$ which have the value zero is the number of the $x$ 's (or $y$ 's) which can be removed by linear transformation. Each $M_{i}$ which exceeds zero will be denoted by $m_{i}$; let their number be $p$. In the notations of Theorem 2, a canonical form is

$$
C=\sum_{i=1}^{p} T_{i}+u \gamma+v \delta, \quad T_{i}=R_{i}+S_{i},
$$

with $n_{i}=m_{i}$ in $S_{i}$, while $u \gamma+v \delta$ is symmetric (or Hermitian), has a determinant not zero identically in $u$ and $v$, and has the same invariant factors as $u \phi+v \psi$. Then the latter is equivalent to $C$ in the sense of Theorem 4.

To emphasize the fact that each $T_{i}$ is symmetric (or Hermitian), write $x_{\alpha}$ for $\xi_{\alpha}, x_{m_{i}+\alpha}$ for $X_{\alpha}, y_{\alpha}$ for $Y_{\alpha}$, and $y_{m_{i}+\alpha}$ for $\eta_{\alpha}$.

TheOREM 5. In the singular case, any pair of symmetric (or Hermitian) bilinear forms can be reduced by a non-singular linear transformation on the $x$ 's and the cogredient (or conjugate) transformation on the $y$ 's to a canonical pair which are the coefficients of $u$ and $v$ in $\sum T_{i}+u \gamma+v \delta$, where

$$
T_{i}=\sum_{\alpha=1}^{m_{i}}\left[\left(u x_{i \alpha-1}+v x_{i \alpha}\right) y_{i m_{i}+\alpha}+\left(u y_{i \alpha-1}+v y_{i \alpha}\right) x_{i m_{i}+\alpha}\right]
$$

is symmetric and Hermitian in its $2 m_{i}+1$ pairs of variables, while $\gamma, \delta$ is a canonical pair for the non-singular case.

*Modern Algebraic Theories, pp. 122-3.

$\dagger$ For the quadratic case, we may employ Weierstrass's canonical pair, Modern Algebraic Theories, p. 131. For the Hermitian case, we may employ the corresponding pair, Logsdon, Ainerican Journal of Mathematics, vol. 44 (1922), p. 254. Or, without separating the two cases we may employ the writer's pair, Modern Algebraic Theories, pp. 126-8. In all of these cases, $\gamma$ and $\delta$ have any prescribed invariant factors (or elementary divisors). 
III. Pairs of quadratic or Hermitian forms in the singular case

Let $f=u \phi+v \psi$ be of type (29). To it corresponds

$$
h=\sum_{i, j=1}^{n} a_{i j} x_{i} \tilde{x}_{i}, \quad a_{i i}=\tilde{a}_{i j},
$$

which is a quadratic or Hermitian form according as $\tilde{a}$ is identified with $a$ or its conjugate imaginary, and likewise for $\tilde{x}$. In the respective cases, write $g=2$ or 1 . Then

$$
\frac{\partial h}{\partial x_{i}}=g \sum_{j=1}^{n} a_{i j} \tilde{x}_{i}, \sum_{i=1}^{n} c_{i} \frac{\partial h}{\partial x_{i}}=g \sum_{j=1}^{n}\left(\sum_{i=1}^{n} c_{i} a_{i j}\right) \tilde{x}_{i}=0,
$$

by (31). Hence any linear relation (30) between the first derivatives of $f$ with respect to $x_{1}, \cdots, x_{n}$, whose coefficients $c_{i}$ are polynomials in $u$ and $v$, implies a linear relation with the same coefficients $c_{i}$ between the first derivatives of $h$, and conversely. Hence the minimal numbers $M_{i}=N_{i}$ of $f$ coincide with those of $h$. By* Theorem 4, we have

THEOREM 6. In the singular case, two pairs of quadratic or Hermitian forms are equivalent if and only if they have the same invariant factors and the same minimal numbers $M_{i}$.

From Theorem 5 we obtain a canonical pair of Hermitian forms by identifying each $y$ with the conjugate imaginary to $x$, and a canonical pair of quadratic forms by identifying each $y$ with $x$. The latter pair is

$$
\gamma+2 \sum_{i=1}^{p} \sum_{\alpha=1}^{m_{i}} x_{i \alpha-1} x_{i m_{i}+\alpha}, \delta+2 \sum_{i=1}^{p} \sum_{\alpha=1}^{m_{i}} x_{i \alpha} x_{i m_{i}+\alpha},
$$

where $\gamma, \delta$ is the canonical pair in the non-singular case (last foot-note in II). In particular, there exists a pair of quadratic or Hermitian forms with any prescribed invariant factors and any prescribed minimal numbers.

An example of (33) is given by $2 x_{0} x_{2}, 2 x_{1} x_{2}$. We have

$$
v \frac{\partial f}{\partial x_{0}}-u \frac{\partial f}{\partial x_{1}} \equiv 0, \quad f=2 u x_{0} x_{2}+2 v x_{1} x_{2} .
$$

Here there is a single minimal number $M_{1}=1$ and no invariant factor.

*And the corollaries on pp. 66 and 68 of Modern Algebruic Thesries.

Untversity of Chicago,

Chicago, Ill. 